\title{
Phase Transitions of Bulk Statistical Copolymers Studied by Dynamic Monte Carlo Simulations
}

\author{
Wenbing Hu, ${ }^{* \dagger}$ Vincent B. F. Mathot, ${ }^{\ddagger}$ and Daan Frenkel ${ }^{\dagger}$ \\ FOM Institute for Atomic and Molecular Physics, Kruislaan 407, \\ 1098 SJ Amsterdam, The Netherlands, and DSM Research, P.O. Box 18 Geleen, The Netherlands \\ Received August 26, 2002
}

\begin{abstract}
We report a numerical study of crystallization and melting in bulk statistical homogeneous (random), homogeneous (slightly alternating), and heterogeneous (produced in a batch reaction) copolymers formed by crystallizable monomers and noncrystallizable comonomers. In our dynamic Monte Carlo simulations of lattice chains, the current model further assumes that the comonomers cannot move into crystalline regions by sliding diffusion of the chains. We find that both the overall composition and the statistical distribution of the monomers affect the phase-transition temperature, the resulting relative crystallinity, and the crystal morphology. However, the final absolute crystallinity of homogeneous copolymers seems insensitive to these parameters. I ntramolecular segregation between monomers and comonomers is accompanied by crystallization, demonstrating the concept of sequence segregation or nanophase separation of statistical copolymers with assembling structures like in thermoplastic elastomers. Moreover, if crystallization of homogeneous copolymers has started but not yet completed on cooling, subsequent heating will show cold crystallization before melting, which can be attributed to insertion-mode lamellar growth. For heterogeneous copolymers, intermolecular segregation occurs on cooling before crystallization. On the basis of our observations, a pair of master melting and crystallization curves for the crystallinity of a statistical copolymer as a function of temperature are suggested to reflect the characteristic of the monomer-sequence-length distribution. This suggestion facilitates the clarification to the kinetic disturbance in local temperature regions and to the principle of some fractionation methods, such as temperature rising elution fractionation (TREF).
\end{abstract}

\section{Introduction}

Crystallization and melting of synthetic polymers are particularly sensitive to the sequence irregularities al ong the chains. The invention of Ziegler-Natta catalysts for the synthesis of polyolefins in the 1950s resulted in improved sequence regularities. This development gave an impetus to the study of crystallization and crystal morphology of semicrystalline polymers. In recent years, metall ocene-based catalysts have enabled even better control of the sequence regularity. This opens the prospect of better control of crystallization and morphology and, as a consequence, the possibility to design polymers with improved properties. However, our present knowledge about the relationships between sequence (ir)regularities and phase-transition behavior is still very limited, even for the most widely studied ethylene-based copolymers. ${ }^{1-3}$

Typically, the sequence (ir)regularities in polymers can have their origin in nonhomogeneous chemical composition, in variations in bond geometries, or in the mixing of stereoenantiomeric monomers. In general, irregular sequences give rise to a different spatial shape than regular sequences and therefore lack the ability of regular sequences to form close-packed structures at a specific temperature. Such packing is the source of the enthalpy gain, i.e., the main driving force for polymer crystallization, which should compensate for the entropy loss during phase transition. If we consider the units of irregular sequences as noncrystallizable comonomers, it is possible to simulate the matching of the units of crystallizable sequences using a microscopic

\footnotetext{
† FOM Institute for Atomic and Molecular Physics.
}

‡ DSM Research. model based on the packing energy difference between monomer pairs and pairs containing comonomers, re sulting in a simulation of the crystallization and melting behavior of copolymers. ${ }^{4,5}$

Early in 1947, Flory suggested an equilibrium theory to predict the melting-point depression due to exclusion of comonomers from the crystallites. ${ }^{6,7}$ This theory was subsequently extended to allow for partial inclusion of comonomers in the crystallites in order to fit the available experimental results. ${ }^{8,9}$ Although the predictions of these equilibrium theories ${ }^{10}$ are qualitatively correct, it is actually impossible to verify the quantitative predictions with the experimental results of copolymers. ${ }^{11}$ First, there is no characteristic melting point for copolymers, since the distribution of sequence lengths gives rise to a distribution of crystallization temperatures, resulting a wide crystallite size distribution and hence, a wide melting temperature range. Second, a true equilibrium distribution of sequence lengths in corre sponding crystallites cannot be approached in reality due to the irreversible nature of polymer crystallization. During copolymer crystallization, the difficulty of longdistance diffusion of molecules makes a complete fractionation of sequence lengths at the crystal-growth front impossible. Moreover, Flory-type theories assume that the crystallizable sequences are fully extended in the crystallites. In practice, however, the resulting crystallites usually exhibit appreciable chain-folding. Only in specific situations, for instance during crystallization of ethylene-based copolymers under high pressure ${ }^{12,13}$ are the long ethylene sequences expected to be more extended in the crystallites.

The difficulty of molecular diffusion is due to both the high viscosity of polymer melts and the restriction that some chain units are incorporated in the present crys- 
tallites. ${ }^{14}$ Moreover, sliding diffusion of copolymers is difficult because the usually large-sized comonomers cannot pass through close-packing regions of monomers. Such close-packing regions can be located in the crystallites or even be generated by thermal fluctuations in the melt. This kind of restriction is quite general for ethylene-based copolymers, and in Flory-type theories, it was simply treated as exclusion of comonomers from the crystallites, disregarding its practical hindrance to approach the extended-chain crystallites with equilibrium distribution of sequence lengths inside. In our simulations, this kind of restriction can be taken into account explicitly in such a way that partial sliding diffusion is possible in the microrelaxation of the lattice chains. ${ }^{15}$

The crystallization and subsequent melting of bulk statistical copolymers with three different kinds of sequence distribution were studied by dynamic Monte Carlo simulations. In describing the sequence distributions, we use the nomenclature for copolymers recommended by the Commission on Macromolecular Nomenclature of the IUPAC Macromolecular Division. ${ }^{16}$ The first kind of sample follows zeroth-order Markovian statistics corresponding to the results of random copolymerization, the second kind of sample fol lows firstorder Markovian statistics with quite different reactivity ratios between monomers and comonomers and a fixed feed composition, corresponding to the homogeneous copolymers produced in a continuous reaction process with a single-site catalyst, and the last kind of sample also follows first-order Markovian statistics with different reactivity ratios between monomers and comonomers but with a significant compositional drift, corresponding to heterogeneous copolymers produced in a batch reaction process with a single-site catalyst. We applied the same cool ing and subsequent heating processes to all copolymers under study and traced the evolution of crystallinity and morphology with temperatures for variable comonomer contents and sequence distributions.

In the remainder of this paper, the principles for the preparation of three kinds of copolymer are described in some details. After that, we give a simple description of the model and the simulation algorithm used. Next, the simulation results are reported, followed by discussion and conclusions.

\section{Simulation Techniques}

2.1. Sequence Statistics of Copolymers. The statistical distribution of monomers in a copolymer is determined by the various monomeric polymerization reactions to form the chains or by the various substitution reactions on the side-group of chains. For the sake of convenience, we focus our attention on statistical copolymers produced in the copolymerization process of free-radical addition with a single-site catalyst, while monomers form the crystallizable regular sequences and comonomers contribute to noncrystallizable irregular sequences. The distribution of reacted (co)monomers along the chains is supposed to follow a Markovian process, ${ }^{16,17,18,19,20}$ which characterizes random copolymers as zeroth order (Bernoullian statistics). In the first-order Markovian process, the propagating probability depends only upon the last unit added to the chain (the terminal model). For the second-order Markovian process (the penultimate model), the last two units incorporated in the chain determines the probability of subsequent (co)monomer addition. Although the penultimate model may fit better to reality at the expense of complexity, ${ }^{21}$ we will use only the first-order Markovian process for simplicity. The propagation reactions of copolymer chains for such a process can be represented using rate constants $k_{i j}$ with $i, j \in\{1,2\}$ where 1 stands for the crystallizable monomer and 2 denotes the noncrystallizable comonomer:

$$
\begin{aligned}
& \sim \sim \sim M_{1}^{*}+M_{1} \stackrel{k_{11}}{\longrightarrow} \sim \sim M_{1} * \\
& \sim \sim \sim M_{1} *+M_{2} \stackrel{k_{12}}{\longrightarrow} \sim \sim \sim M_{2}^{*} \\
& \sim \sim \sim M_{2} *+M_{1} \stackrel{k_{21}}{\longrightarrow} \sim \sim \sim M_{1} * \\
& \sim \sim \sim M_{2} *+M_{2} \stackrel{k_{22}}{\longrightarrow} \sim \sim M_{2} *
\end{aligned}
$$

Here, $\mathrm{M}_{\mathrm{i}}{ }^{*}$ with $\mathrm{i} \in\{1,2\}$ represents the concentration of propagating chains with the end species $i$, and $M_{i}$ with $i \in\{1$, 2 \} the concentrations of (co)monomer i. The corresponding propagation probabilities are then defined as

$$
\begin{gathered}
\mathrm{P}_{11} \equiv \mathrm{k}_{11} \mathrm{M}_{1} \mathrm{M}_{1} * /\left(\mathrm{k}_{11} \mathrm{M}_{1} \mathrm{M}_{1}^{*}+\mathrm{k}_{12} \mathrm{M}_{2} \mathrm{M}_{1} *\right)=\mathrm{r}_{1} \mathrm{~F} /\left(\mathrm{r}_{1} \mathrm{~F}\right. \\
\mathrm{P}_{12}=1-\mathrm{P}_{11} \\
\mathrm{P}_{22} \equiv \mathrm{k}_{22} \mathrm{M}_{2} \mathrm{M}_{2} * /\left(\mathrm{k}_{22} \mathrm{M}_{2} \mathrm{M}_{2} *+\mathrm{k}_{21} \mathrm{M}_{1} \mathrm{M}_{2}{ }^{*}\right)=\mathrm{r}_{2} /\left(\mathrm{r}_{2}+\mathrm{F}\right) \\
\mathrm{P}_{21}=1-\mathrm{P}_{22}
\end{gathered}
$$

where $r_{1} \equiv k_{11} / k_{12}$ and $r_{2} \equiv k_{22} / k_{21}$ are the (co)monomerreactivity ratios, and $F \equiv M_{1} / M_{2}$ is the feed mole-fraction ratio of monomer and comonomer. In this modeling process, the polymerization kinetics can be fully accounted for by one set of $r_{1}$ and $r_{2}$ (characterizing the catalyst) plus the feed composition $\mathrm{F}$. In this way, it is straightforward to model the sequence distribution of statistical copolymers. ${ }^{22}$

The product of $r_{1}$ and $r_{2}$ determines the nature of the sequence distributions. If $r_{1} r_{2} \gg 1$, cross propagation will be unlikely and a copolymer with long blocks will result. If $r_{1} r_{2}$ $\ll 1$, cross propagation will be dominant, thus favoring alternating copolymer sequences. Most of the practical copolymers turn out to have statistics between alternating and random, i.e., $0<r_{1} r_{2}<1$.

Copolymerization is typically done in a continuous reaction process with a single-site catalyst, so there will be no difference between the chains with respect to statistics of (co)monomer incorporation: there is both intra- and intermolecular homogeneity. Such statistical copolymers are usually called homogeneous. ${ }^{3,23}$ If the propagation probabilities that determine the composition of the chains are not constant either within one chain or from one chain to the next, the copolymer may be a mixture of chains with quite different comonomer contents and sequence distributions. In such cases, the copolymer is said to be heterogeneous. An example is a batch reaction process with a single-site catalyst. If the reactivity ratios are very different, then there will be an appreciable change in feed composition as the reaction proceeds. This compositional drift is avoided in continuous reaction processes, and the heterogeneity of most copolymers is caused by the multisite nature of catalysts, i.e., multiple r-sets. This is, for instance, the case for linear low-density polyethylenes (LLDPEs) produced with heterogeneous-type Ziegler-Natta catalysts. ${ }^{23-26}$ The implications of the latter source of heterogeneity in copolymers will be reported in the near future. ${ }^{27}$

2.2. Sample Preparation. We produced three kinds of copolymer with sequence statistics corresponding to the abovementioned homogeneous (random), homogeneous (alternatingto-random), and heterogeneous (produced in a batch reaction) copolymers and, for each kind, samples having different comonomer contents. In a cubic lattice box with its linear size of 64, we placed 1920 chains, each chain occupying 128 consecutive sites, so the density of the samples is as high as 0.9375. I nitially, we marked a long, single macromolecule for all the chains. Along this single macromolecule, the first site is label ed as being a monomer (denoted as 1 ) or a comonomer (denoted as 2) according to the probability determined by the 
Table 1. Number of Crystallizable Monomer Bonds in the Three Kinds of Copolymers

\begin{tabular}{|c|c|c|c|c|}
\hline \multirow{2}{*}{$\begin{array}{l}\text { comonomer } \\
\text { mole } \\
\text { fraction }\end{array}$} & \multirow{2}{*}{$\begin{array}{l}\text { random } \\
\text { monomer } \\
\text { bonds }\end{array}$} & \multicolumn{2}{|c|}{ slightly alternating } & \multirow[b]{2}{*}{$\begin{array}{l}\text { heterogeneous } \\
\text { monomer bonds }\end{array}$} \\
\hline & & F set & $\begin{array}{l}\text { monomer } \\
\text { bonds }\end{array}$ & \\
\hline 0.06 & 215391 & 0.610 & 215030 & 226015 \\
\hline 0.12 & 188462 & 0.275 & 187041 & 208759 \\
\hline 0.24 & 140863 & 0.108 & 134806 & 175529 \\
\hline 0.36 & 99862 & 0.0541 & 89679 & 143174 \\
\hline 0.44 & 76506 & 0.0356 & 63563 & 122111 \\
\hline
\end{tabular}

specified composition, the next site and so on are labeled according to the specified propagation probabilities. A series of samples for each kind of copolymer were then generated with comonomer mole fractions of $0.06,0.12,0.24,0.36$ and 0.44 , respectively.

For the homogeneous random copolymers, $r_{1}=r_{2}=1$, and $\mathrm{F}$ is a constant calculated from a preset comonomer content. For simplicity in the following text, we denote this series of samples as random copolymers. For the homogeneous alternating-to-random copolymers, $r_{1}=24.7$ and $r_{2}=0.017$ were taken in analogy to the copolymerization of ethene and 1-octene using a vanadium-based Ziegler-Natta catalyst. ${ }^{3}$ The value of $F$ is chosen to be consistent with the specified content of comonomers in the final product (see Table 1). Since $r_{1} r_{2}=$ 0.42 , the sequence distribution of this type of homogeneous copolymers will reflect a tendency toward alternating. For simplicity in the following text, we name this series of samples as slightly alternating copolymers.

F or the heterogeneous batch-reaction-produced copolymers, $r_{1}=24.7, r_{2}=0.017$, but $F$ defined by the (co)monomers in the unreacted residue is not constant, while the preset total composition is fixed. Since the monomers have the highest chance in reaction, the feed monomers are depleted quickly, and chains containing high comonomer content will be generated subsequently. We refer to this series of samples as heterogeneous copol ymers.

In our model, two consecutive monomers along the chain form a monomer bond, and only the interactions between adjacent-but-nonbound monomer bonds contribute to the enthalpy gain. The number of crystallizable monomer bonds in a copolymer changes with the sequence distribution, even though the comonomer content may be constant. Table 1 lists the total number of monomer bonds for samples representing three kinds of copolymer, which are useful in the calculation of absolute crystallinities as defined later on.

Figure la shows the probability distribution of monomersequence lengths for three kinds of copolymer, all with a comonomer content of 0.44 . F or the homogeneous copolymers, the distribution agrees with the theoretical prediction that the probability to generate a monomer-sequence length of $n$ is given by $P_{21} P_{11}{ }^{n-1} P_{12}$ for $n>0$ and by $P_{22}$ for $n=0$. The very long sequences are lost when we split the long single macromolecule into short chains. However in our cases, their number is so small that the distribution is not affected much, as demonstrated in Figure la. The most probable sequence length of the slightly alternating copolymers corresponds to $n=1$ rather than $n=0$ due to the preference for alternating. This preference causes the slightly alternating copolymer to contain fewer long monomer sequences and, as a consequence, fewer crystallizable monomer bonds than the random copolymer at the same comonomer content. However, the heterogeneous copolymer contains a significant number of both the longest and the shortest monomer sequences.

Figure $1 b$ shows the population distribution of chains that have a specified number of comonomer units at constant overall comonomer fraction 0.44 . The figure shows that the random and the slightly alternating copolymers have a singlepeaked distribution while the heterogeneous copolymer exhibits a double-peaked distribution. For heterogeneous copolymers, the number of chains consisting exclusively of comonomers is about 660 , i.e., close to one-third of the total number of chains (1920). Hence, this system can be regarded as a blend of crystallizable and noncrystallizable chains.
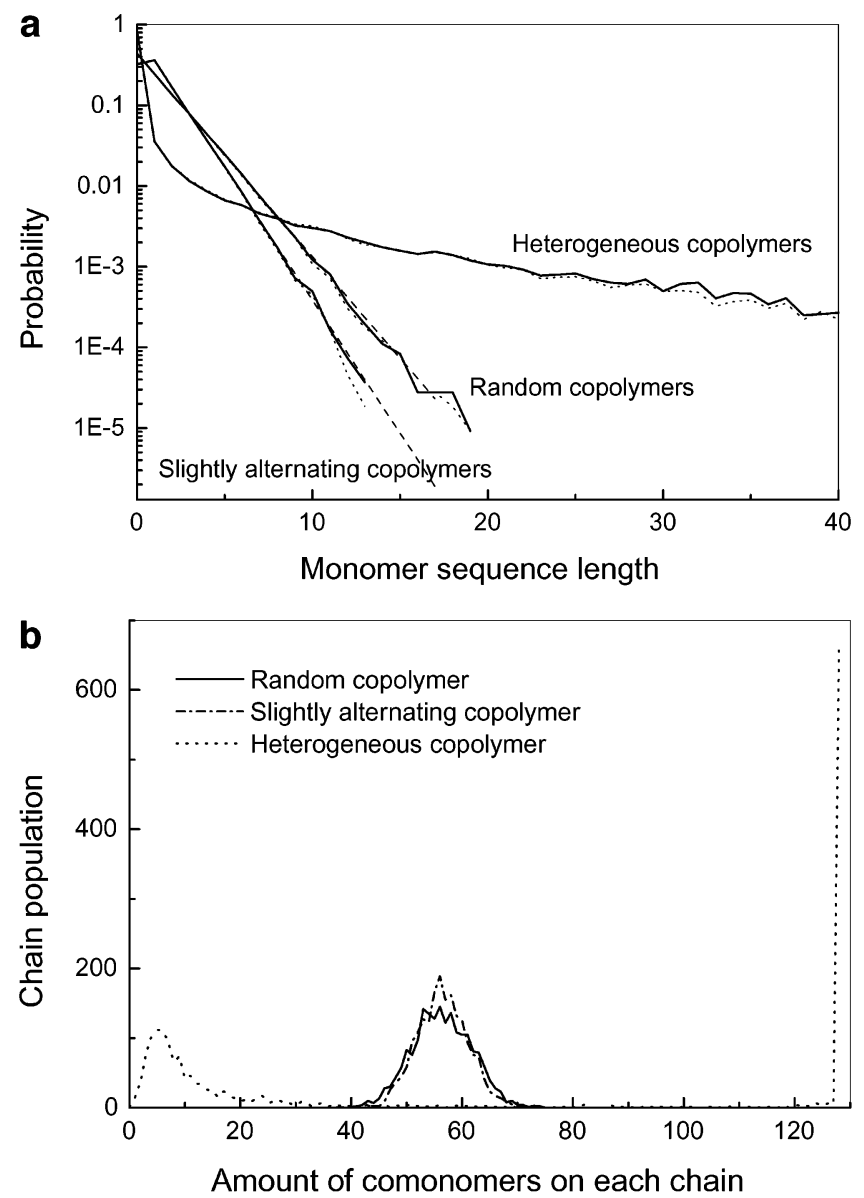

Figure 1. Microstructure characterizations of random, slightly alternating, and heterogeneous copol ymers with a comonomer mole fraction of 0.44 . (a) Number distributions of monomersequence lengths. The solid lines are for the long single macromolecule, the dotted lines are for the chains resulting after splitting the single macromolecule, and the dashed lines are the theoretical predictions (see text). (b) Chain distribution according to the number of comonomer units in the chain. Only the segments connecting the data points are drawn for clarity.

2.3. Microrelaxation Model and Algorithm. The Monte Carlo scheme that we use to simulate polymer motion belongs to the class of single-site jumping models that was pioneer ed by Larson et al. ${ }^{28}$ Larson et al. considered models based on kink generation and end-to-end sliding-diffusion. The kinkgeneration scheme was subsequently devel oped into the bondfluctuation model. ${ }^{29,30}$ A hybrid model that combines kink generation with the sliding diffusion was suggested by $L u$ and Yang. ${ }^{31}$ In this scheme, it is assumed that sliding diffusion can start in the middle of the chain but finish at a chain end. In our hybrid model, based on de Gennes's original picture of defect diffusion along a chain, ${ }^{32}$ we assume that the region of sliding diffusion can be terminated by decreasing the sharpness of the kink conformation in the middle of the chain. ${ }^{15}$

Kinks can only be generated if the required void space is locally available. To enhance the efficiency of our simulation algorithm in dense systems, we first randomly select one of the small number of void sites. Next, we randomly select a site adjacent to this void. If this site is occupied by a chain unit, then a microrelaxation step is attempted.

In our simulations, we use periodic boundary conditions. We use a cubic box shape, but this is not essential. A lattice site can be occupied by one monomer at most, and polymer chains cannot cross to each other. I nter-monomer bonds are possible either along six lattice directions or al ong 12 face diagonals and eight body diagonals. Hence, the coordination number of a monomer is $6+12+8=26$. 
In the present simulations, we also have to consider the effect of the comonomers on the sliding diffusion. In particular, we have to implement a rule that takes into account that comonomers cannot diffuse into crystalline regions. This we do as follows: if a comonomer were to move along a bond previously connecting two monomers, we test whether this bond has more than one neighboring parallel bond. If so, the region is considered locally crystalline, and the trial move is rejected.

To initiate our simulations, the chains were placed regularly on the lattice. During equilibration, the chains are allowed to relax to the coil state. This equilibration is performed at high temperatures and without any restriction on sliding diffusion. After that, we make a distinction between monomer and comonomer units. The system is cooled, and the dynamics proceeds by microrelaxation steps with sliding diffusion, including the constraints on comonomer diffusion.

Moves that are not rejected because of hard-core overlap or bond crossing can result in a change of the potential energy due to a change in the number of parallel bonds. This leads to a potential-energy change $p E_{p}$, where $p$ is the decrease in the number of neighboring parallel bonds for those monomers jumping and releasing a void site. In addition, a potentialenergy penalty for chain bending and the potential-energy exchange between the pairs of monomer and those of comonomer could have been included. ${ }^{5,33}$ However, for the sake of simplicity, we supposed that the chains are fully flexible and that the mixing between monomers and comonomers is athermal. Trial moves are accepted with a probability given by the Metropolis criterion.

2.4. Temperature Scanning Programs. In analogy to a differential scanning calorimetry (DSC) measurement, we stepwise changed the reduced temperature $k_{B} T / E_{p}$ in steps of size 0.01. During each step, we carried out 300 Monte Carlo (MC) cycles, where one MC cycle is defined as one trial move per (co)monomer averaged over the sample system. The first 100 MC (equilibration) cycles are discarded. Averages were computed during the subsequent $200 \mathrm{MC}$ cycles. The molten chains were first cooled from the reduced temperature of 6 to 2 or to 1 . In this temperature range, crystallization is expected to occur. Then, the crystallized samples were heated from the lower temperatures back up to the initial temperature, where the melting is expected to occur. We compared two heating curves with different depths of cooling for the random and the slightly alternating copolymers.

In addition, we performed a cooling process with a stepwise increase in the value of the reduced reciprocal temperature $\mathrm{E}_{\mathrm{p}} /\left(\mathrm{k}_{\mathrm{B}} \mathrm{T}\right)$ starting from zero (infinitely high temperature), to check out possible segregation between two species of chain units above the crystallization temperature.

2.5. Traced Quantities. In the crystalline region, the maximum of crystallizable monomer bonds packing in parallel around one monomer bond can be 24 , which is the coordination number of 26 minus two intrachain bonds. Meanwhile in the amorphous region, the amount of parallel packing is usually found to be close to zero. To distinguish disordered regions from (partly) crystalline regions, we used a criterion of 5 that would effectively identify the amorphous state and the crystalline state, even when defect-rich. During the temperature scanning, the crystallinity was therefore defined as the fraction of monomer bonds, which contain more than five neighboring parallel monomer bonds, in the total amount of monomer bonds. This crystallinity is not equal to the total crystallinity, which is the fraction in the total amount of bonds without distinction in composition of monomers and comonomers, i.e., the sample mass. Nor does it represent the relativecrystallinity where the denominator is the number of monomers, rather than the number of monomer bonds. One can view the present definition of crystallinity as a measure of the absolute crystallinity, since it reflects the fraction of maximum attainable crystallizability. In contrast, experiments only report the results by means of the total crystallinity or the relative crystallinity.

The (de)mixing of monomers and comonomers was traced by evaluating the mean fraction of neighboring sites of each


Figure 2. Relative crystallinities (a) and absolute crystallinities (b) (see definitions in text) as a function of reduced temperature on cooling and subsequent heating (see arrows) for a series of random copolymers with variable comonomer mole fractions as denoted. The solid curves are for cooling from 6 to 1 , the dashed curves are for heating starting from 2, and the dotted curves are for heating starting from 1 .

comonomer occupied by other comonomers. This quantity, which can be called as the demixing parameter of comonomers, is sensitive to changes in the local environment of the comonomers due to any composition segregations.

\section{Results}

3.1. Random Copolymers. Figure 2 shows the curves of the relative crystallinity (a) and the absolute crystallinity (b) for the series of random copolymers on cooling and subsequent heating. The significant hysteresis between heating and cooling curves reflects nucleation-controlled first-order phase transition on cooling. Before major crystallization sets in, the melt may already have an appreciable level of crystallinity due to local fluctuations. The onset of phase transitions is found to be very sensitive to the comonomer content. This is, in fact, well-known from experiments: increasing the comonomer content moves the crystallization and melting transitions to lower temperatures. Also, the relative crystallinity on cooling follows the sequence of comonomer content, which is consistent with the experimental observations, such as those on homogeneous ethylene-1-octene copol ymers. ${ }^{34}$ However, the absol ute crystallinities saturate at around 0.8 . This saturation value seems insensitive to the comonomer content. The only exception is the pure homopolymer, which shows a higher crystallinity (0.9). It seems likely that the 
a

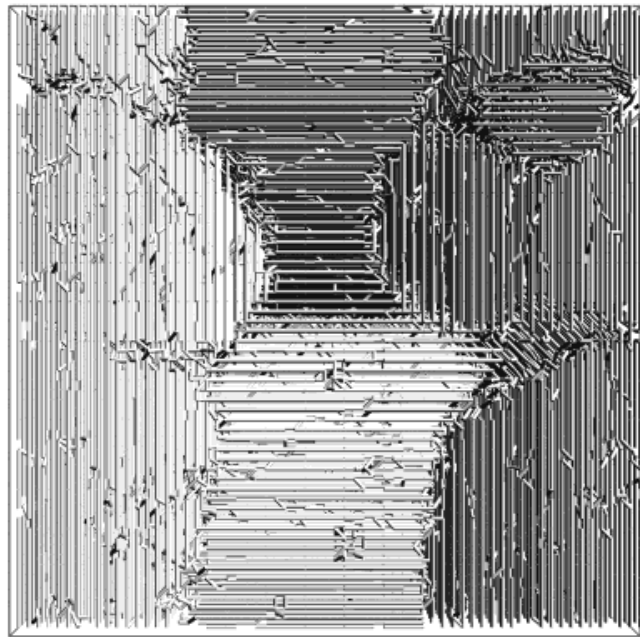

C

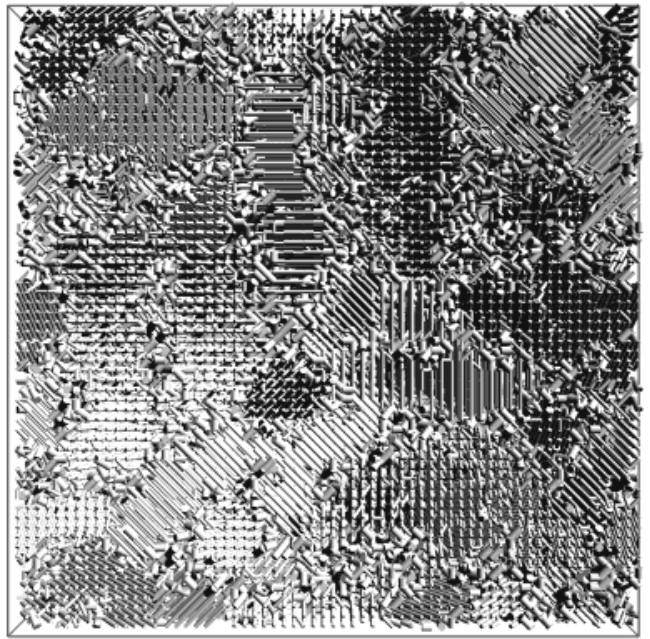

e

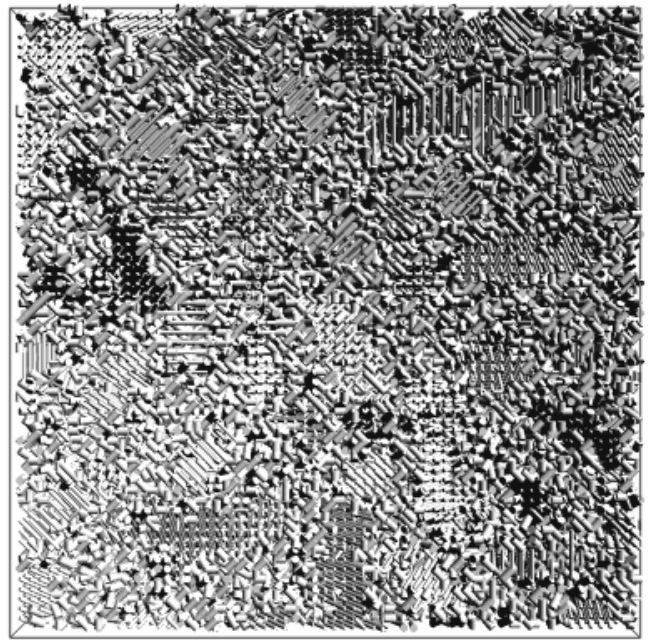

b

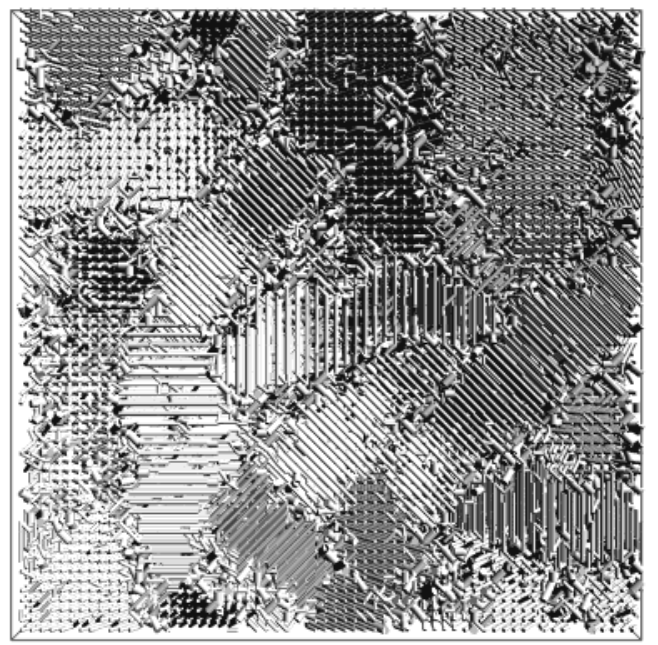

d

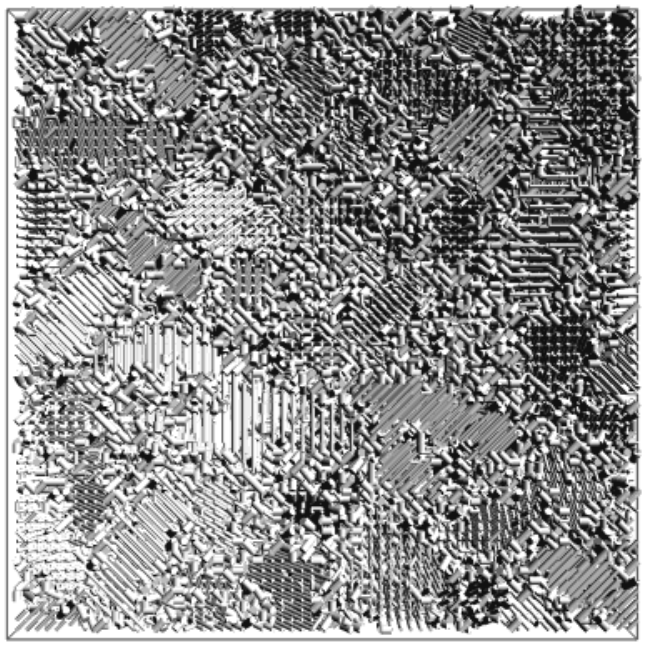

f

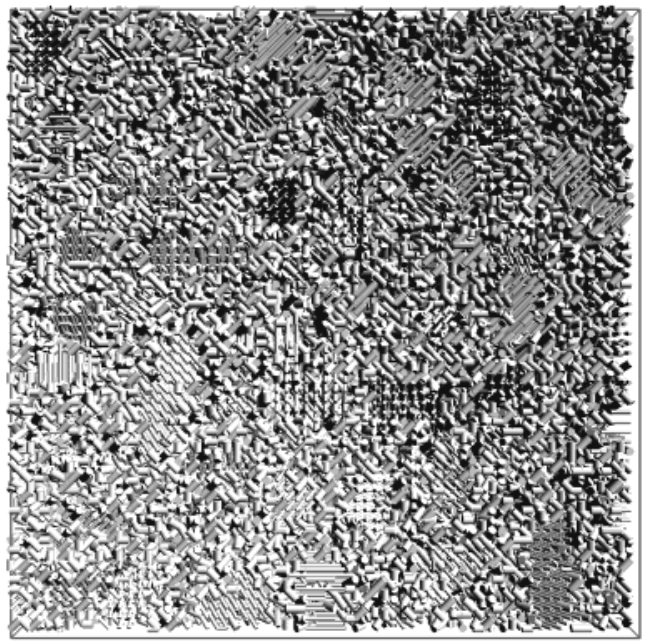

Figure 3. Snapshots of random copolymers with variable comonomer mole fractions in a cubic lattice with periodic boundary conditions at the reduced temperature of 1 . Parts a-f are for the comonomer content of $0.0,0.06,0.12,0.24,0.36$, and 0.44 respectively. The polymer bonds are drawn in cylinders, and the bonds containing comonomer units have double thickness.

amorphous monomer bonds are situated at the surface of metastable crystallites. F or copolymers, more metastable crystallites are generated than in homopolymers. This may explain why the crystallinity of homopolymers saturates at a higher value.

In Figure $2 \mathrm{~b}$, when the comonomer content is high and the crystallinity is still far from maximum at the reduced temperature of 2 , the heating curves starting from 2 show significant cold crystallization before melt- ing. If the comonomer content is not high, the heating curves with different starting points overlap almost completely. Since the melting temperatures mainly depend on the size and perfection of the polymer crystallites, this observation implies that those crystallites that melt in the overlapping temperature region are not annealing as they are heated. The dependence of the heating curves on the initial temperature, which is observed for systems with a high comonomer content, 

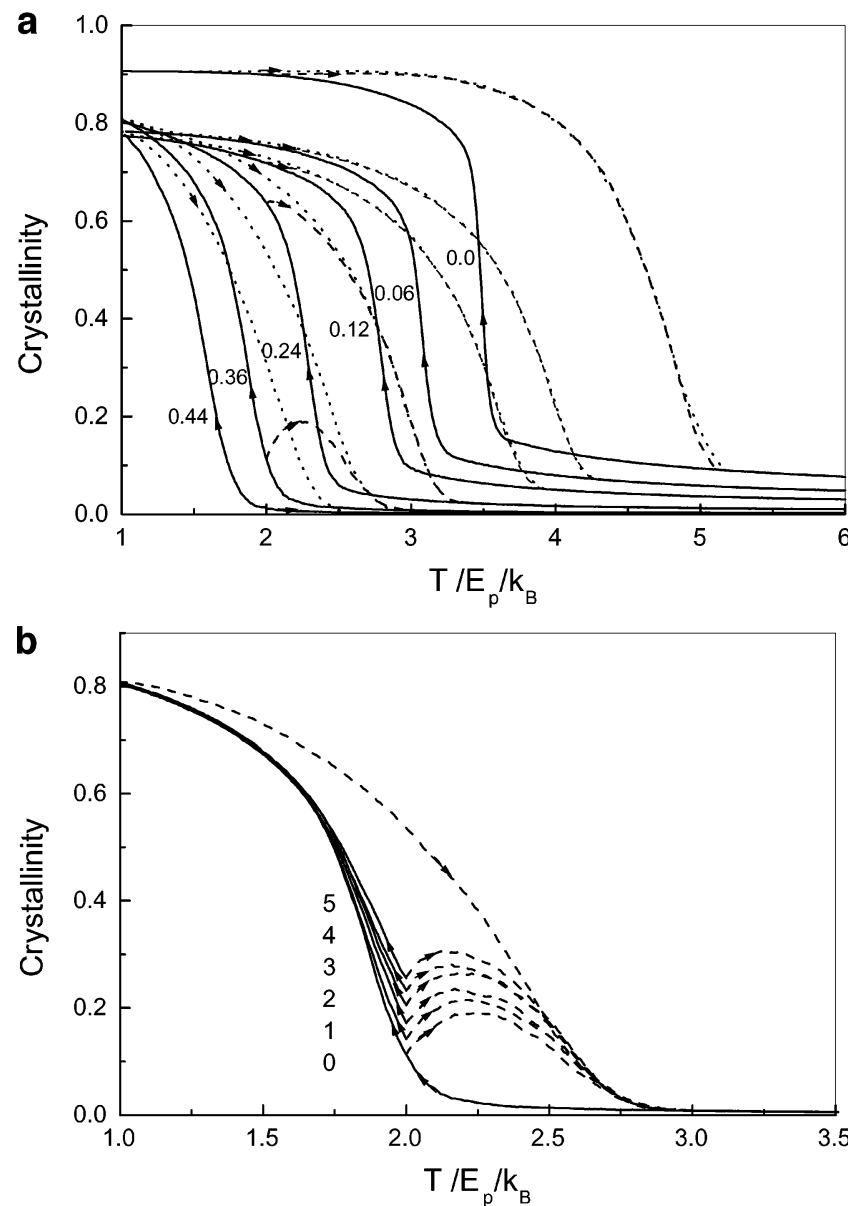

Figure 4. Absolute crystallinities as a function of reduced temperature on cooling and subsequent heating (see arrows): (a) for a series of slightly alternating copolymers with variable comonomer mole fractions as denoted; (b) for the slightly al ternating copolymer with a comonomer content of 0.36 after annealing at the reduced temperature of 2 for variable periods of time as denoted ( $\times 1000 \mathrm{MC}$ cycles). The solid curves are for cooling, the dashed curves are for heating starting from 2 , and the dotted curve is for heating starting from 1.

must be due to the difference in the thermal history of smaller (less stable) crystallites.

Figure 3 shows the crystal morphologies of the series of random copolymers at a reduced temperature of 1 . Figure $3 a$ demonstrates that in a homopolymer system, sliding diffusion of chains tends to thicken the initially lamellar crystallites. ${ }^{33}$ As a consequence, the crystallinity can be as high as 0.9. Figure $3 \mathrm{~b}$ demonstrates that just a small number of comonomers (mole fraction 0.06) significantly change the crystal morphology. The lamellae are rather thin and it seems that further thickening is inhibited by the presence of comonomers on the fold surface. This stability of the crystallite thickness has been observed in small-angle X-ray scattering (SAXS) experiments on syndiotactic polypropylene-1-octenes. ${ }^{35}$ The well-devel oped lateral sizes of the lamellae imply an apparent amount of chain folding in the crystallites in order to avoid steric crowding of dangling ends at the fold surfaces. At high comonomer content, Figure 3 shows small crystallites. The crystallites for the copolymer with a comonomer content of 0.44 appear to have granular texture, similar to the experimental observations on ethylene-based copolymers. ${ }^{36}$ The deterioration of the crystallite morphology with the increase of comonomer content is consistent with the experimental observations dating back to Kilian and Fischer ${ }^{37}$ and

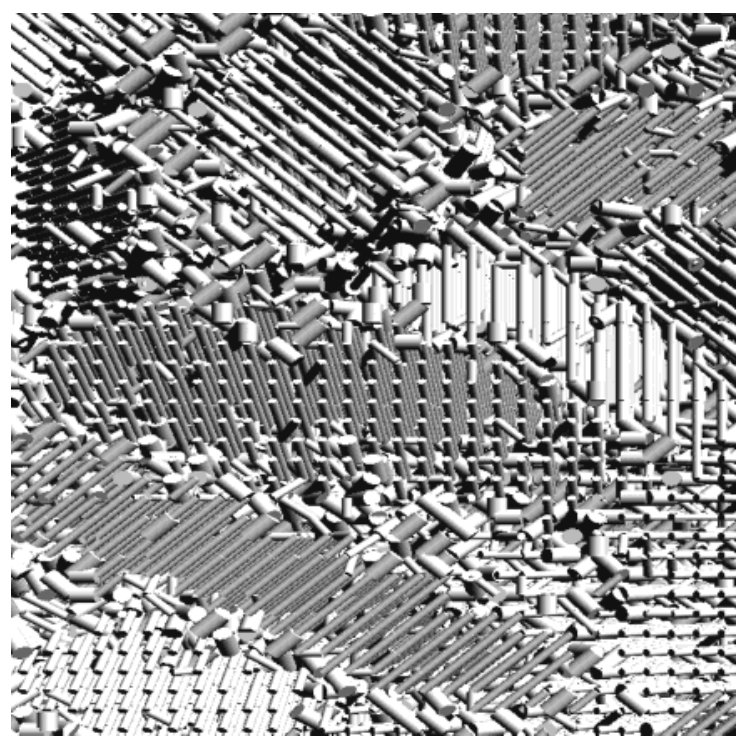

Figure 5. Magnified partial region in a snapshot of the slightly alternating copolymer with comonomer mole fraction 0.12 . The polymer bonds are drawn in cylinders, and the bonds containing comonomer units have double thickness.

also with the simulations ${ }^{4}$ for homogeneous ethylenepropylene copolymers.

However, the granular crystallites can be observed even in Figure 3b, coexisting with the lamellar crystallites. This coexistence of different kinds of morphology has already been observed in experiments. $3,38-40$

3.2. Slightly Alternating Copolymers. Figure $4 a$ shows the evolution of the absolute crystallinity of the slightly alternating copolymers on cooling and subsequent heating. As expected on the basis of the monomersequence-length distribution, the phase-transition temperatures are slightly lower than those for the corresponding random copolymers, in particular for the samples with high comonomer content. However, the saturation value of the absolute crystallinity on cooling is al most the same as in the case of random copolymers. Heating curves with different starting points are seen to merge in the later stage of melting, as is the case for random copolymers.

For the slightly alternating copolymer with a comonomer content of 0.44 , cold crystallization does not occur when the heating starts from 2 . Probably because, on cooling crystallization has not started yet at this temperature though the melt is supercooled. This implies that cold crystallization only happens if the system contains some crystallites.

Figure $4 \mathrm{~b}$ shows that if the sample with a comonomer content of 0.36 is stopped on cooling at the reduced temperature of 2 and then be annealed for variable periods of time, the crystallinity curves eventually merge both on heating and on further cooling. This demonstrates that annealing disturbs both heating and cool ing crystallinity curves only locally. This observation is consistent with recent DSC measurements on ethylene-1-octene copolymers ${ }^{39,41}$ and reveals a kind of master curve irrespective of Iocal disturbances caused by the scanning rate or the isothermal annealing. It seems logical to conclude that, on cooling, long se quences crystallize earlier forming large crystallites that remelt only at higher temperatures. Short sequences crystallize at lower temperatures and form small crystallites that remelt earlier. Depending on the sequencelength distribution determined by the statistics of 


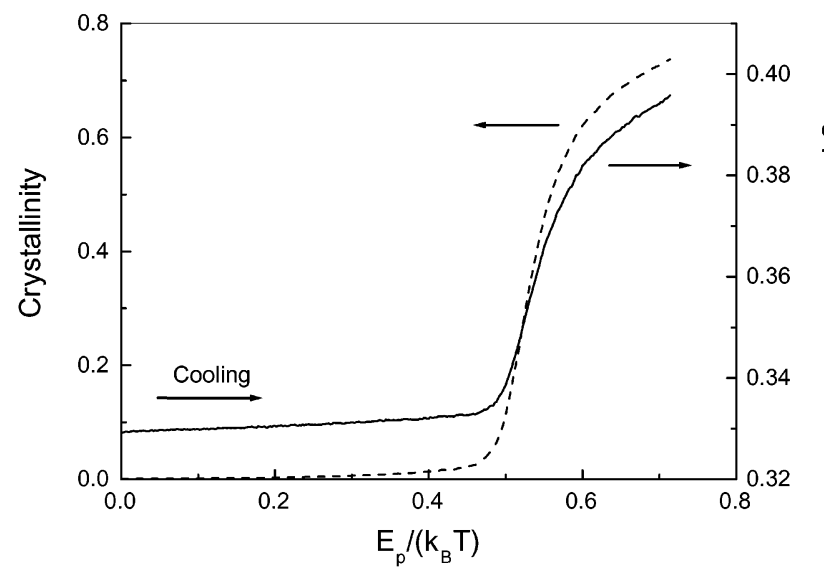

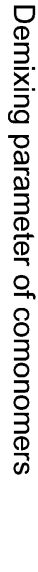

Figure 6. Cooling curves of the absolute crystallinity (dashed line) and the demixing parameter of comonomers (solid line, see the definition in text) for the slightly alternating copolymer with a comonomer content of 0.36 , upon cooling from the infinite temperature and with a stepwise increase in the value of $E_{p} /\left(k_{B} T\right)$ starting from zero with a step length of 0.002 and a step period of $300 \mathrm{MC}$ cycles.

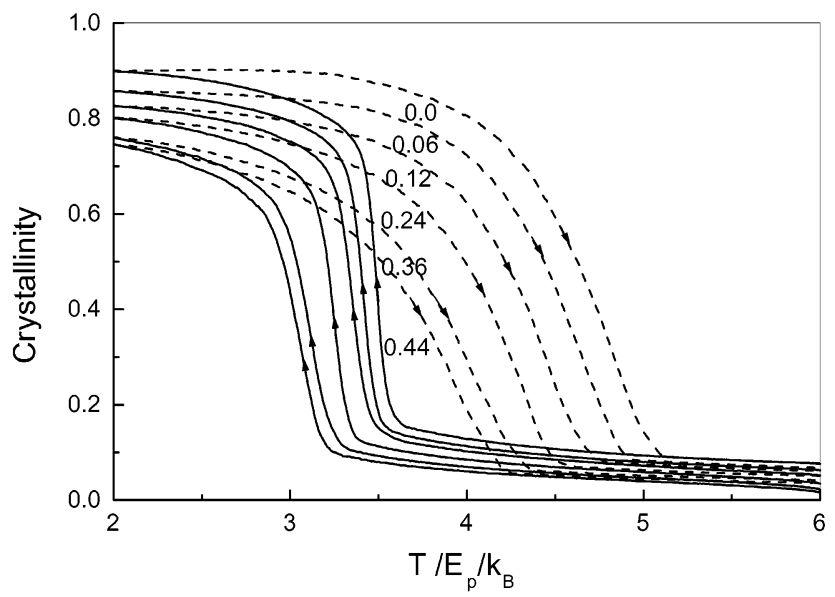

Figure 7. Absolute crystallinities as a function of the reduced temperature on cooling and subsequent heating (see arrows) for a series of heterogeneous copol ymers with variable overall comonomer mole fractions as denoted. The sequence of chains in the sample system was randomized at the initial state before cooling. The solid curves are for cooling from 6 to 2, and the dashed curves are for heating from 2 to 6 .

(co)monomer incorporation during polymerization, there should exist a pair of master cool ing and heating curves for each statistical copolymer. Differences with respect to the depth of cooling, the cooling rate, and the annealing temperature and period of time, just give rise to local deviations from these master curves.

The crystallite morphologies of slightly alternating copolymers at the reduced temperature of 1 are quite similar to those shown in Figure 3. Figure 5 shows an enlarged part of a snapshot for a slightly alternating copolymer with a comonomer content of 0.12 . Since the bonds containing noncrystallizable comonomers are drawn in double thickness, one can recognize that comonomers tend to accumulate in the regions around the crystallites. This is actually an illustration of sequence segregation during the crystallization of copolymers. ${ }^{42}$

To make sure that sequence segregation does not occur before crystallization on cooling, we performed cooling process from infinite temperature (see section 2.4). Figure 6 shows the cool ing curves for the demixing a

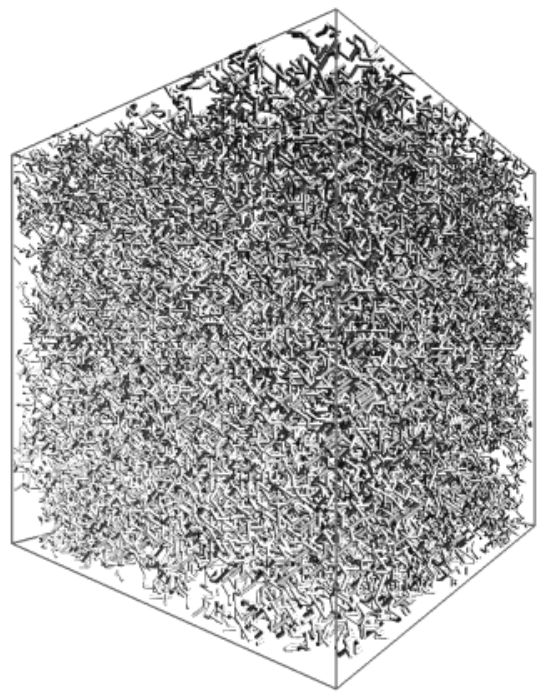

b

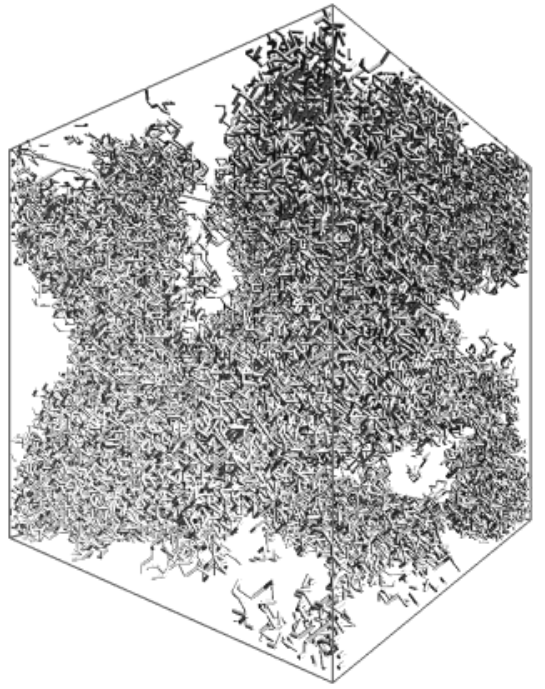

Figure 8. Snapshots of the heterogeneous copolymer with a comonomer mole fraction of 0.36 in the cubic lattice box. Only the chains containing more than 64 comonomers are drawn. Key: (a) snapshot at the initial state of cooling in Figure 7 with randomization of the sequence of chains; (b) snapshot at the reduced temperature of 3.5 in Figure 7.

parameter of comonomers and for the crystallinity in slightly alternating copolymers with a comonomer content of 0.36 . The figure clearly demonstrates that the crystallization is accompanied by the segregation of monomer sequences in homogeneous copolymers.

3.3. Heterogeneous Copolymers. The similar cooling and subsequent heating programs were applied to heterogeneous copolymers. Since the highly crystallizable chains contain a very small amount of comonomers, the phase-transition temperatures are significantly higher than for two homogeneous types of copolymer, but there is still the same dependence of the freezing/ melting on comonomer content (see Figure 7). The crystallization temperatures are so high that the crystallinities are almost saturated at a reduced temperature of 2 and further cooling does not have much effect. The change in crystal morphology with increasing comonomer content is not significant in heterogeneous copolymers. At a reduced temperature of 2 , welldevel oped lamellae can be observed even for a comonomer content of 0.44 . In addition, the ultimate crystallinity of heterogeneous copolymers seems sensitive to 


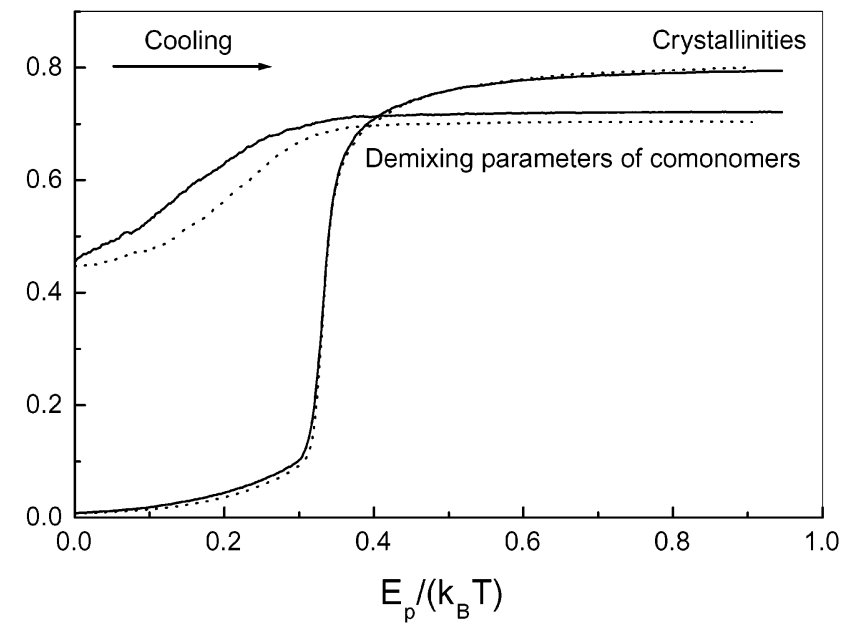

Figure 9. Cooling curves of the absol ute crystal linity and the demixing parameter of comonomers as denoted for the heterogeneous copolymer in Figure 8 with (solid lines) and without (dotted lines) the hard restriction of sliding diffusion of comonomers in the crystalline regions. The cooling program is the same as in Figure 6.

the comonomer content. The observation that heterogeneous copol ymers melt at a higher temperature than homogeneous copolymers with the same composition, agrees with the experimental observations on ethylenebutene copolymers, ${ }^{43,44}$ ethylene-1-octene copolymers, ${ }^{45}$ and other LLDPEs. ${ }^{46}$

The heterogeneous copolymer can be regarded as a blend of copolymers, i.e., partial molecules containing high comonomer content and the other containing low comonomer content. By tracing the morphology of those chains containing high comonomer content, we find that, in a well-mixed heterogeneous copolymer system, molecular segregation occurs on cooling, even before crystallization (see Figure 8). This observation is in agreement with the findings of recent small-angle neutron scattering (SANS) measurements on heterogeneous LLDPEs in a comparison with those on homogeneous copolymers. ${ }^{47}$

To verify the occurrence of molecular segregation in the melt before crystallization, we computed the demixing parameter of comonomers upon cooling from the infinite temperature for the heterogeneous copolymer with a comonomer content of 0.36 . The results are shown in Figure 9. The comonomer demixing parameter curves clearly indicate a trend toward molecular segregation beforecrystallization. If we release the restriction on the sliding diffusion of comonomers in the crystalline region, the segregation transition becomes slightly sharper (see Figure 9), while the subsequent crystallization is not influenced. Furthermore, if the system is reheated, we observe remixing of the polymers even with this diffusion restriction, indicating that this transition is due to thermodynamics rather than kinetics.

\section{Discussion}

In our simulations, we demonstrate that the comonomer content generally lowers the crystallization temperature as well as the melting temperature. In actual homogeneous copolymer systems, the content of comonomers lowers the crystallization temperature $\mathrm{e}^{48}$ and can be so high that the crystallization temperature may shift even below the glass transition temperature, then, only an amorphous state can be realized on cooling. ${ }^{34,49}$ Such copol ymers are usually named noncrystalline pol ymers or amorphous polymers. It has to be remarked that, in our simulations, no such vitrification is included, which is why the crystallinity curves can always go to high values on cooling even for those copolymers with high comonomer content, while in reality, they are usually intercepted by the glass transition.

Comparing three kinds of statistical copolymer in our simulations, it becomes clear that the bulk phase transitions of copolymers are sensitive not only to the comonomer content but also to their distributions within the molecules, in agreement with recent experimental observations $\mathrm{s}^{50}$ and single-chain molecular dynamic simulations. ${ }^{51}$ Difference with respect to sequence distribution (see Figure la) between our random copolymers and slightly alternating copolymers contributes to differences with respect to phase-transition temperatures (see Figures $2 \mathrm{~b}$ and $4 \mathrm{a}$ ). With the same comonomer content, more and longer monomer sequences favor higher crystallization temperatures, Iarger crystallites, and hence higher melting temperatures. This tendency becomes extremely manifest for the heterogeneous copolymers.

The above tendency suggests a pair of master crystallinity curves for crystallization and melting of each statistical copolymer in correspondence to a wide sequence-length distribution. Actually, the idea of this correspondence has been widely applied to explain the multiple melting peaks of DSC curves for commercial LLDPEs, dating back to Mathot $^{23}$ and the temperature correspondence of crystallization to melting for those fractionated LLDPES. ${ }^{52}$ Furthermore, it can even be applied to intrachain heterogeneity of sequence distributions. ${ }^{53}$ Deviations from these master curves can be attributed mostly to kinetic effects, for instance, cocrystallization of monomer sequences of quite different lengths, arising from quenching experiments and extensive recrystallization during subsequent heating of ethylene-1-octene copolymers. ${ }^{54}$

With the increase of comonomer content for those homogeneous copolymers, the morphol ogical deterioration of crystallites is quite significant. In experiments, the large-scale aggregation of crystallites can be observed to change from spherulites to dispersed granular domains. 3,38,39,55 Clearly, the sizes of crystallites are limited by the monomer sequence lengths. Long monomer sequences can form well-developed lamellar crystallites with chain folding. As the lateral dimensions of lamellae decrease with increasing comonomer content or diffusion difficulty at the lower temperatures, granular crystallites result. Then, chain folding becomes unnecessary since crowding does not pose any problem on account of the very small lateral sizes of crystallite. In this case, it is also speculated that fringed micelles result. ${ }^{56}$ Clusters of loosely packed monomer sequences are thought to represent a final state having still some order.

Since the absol ute crystal linity real ized at the lowest temperatures (see Figures $2 b$ and $4 a$ ) is not sensitive to the comonomer content in the case of homogeneous copolymers, all of the monomer sequences seem to have the same chance to be finally included into (poor) crystalline regions. During cooling, the thermodynamic segregation according to the sequence length might be true in the local amorphous regions; 57 i.e., there may exist a local thermodynamic segregation according to the sequence length. ${ }^{57}$ This might be true in the local amorphous regions, where short-range diffusion, even 
at the lowest temperatures, facilitates sequence selection for crystal growth. This is similar to the case of cold crystallization where only the nearest neighbors perform crystallization. ${ }^{58}$ Therefore, different kinds of morphol ogies can coexist in the same copolymer system, especially with middle comonomer content and wide distribution of sequence lengths. A sequential bimodel kinetics is hel pful to clarify the description. ${ }^{59}$ According to the master melting and crystallization curves, long sequences will crystallize first on cooling, with significant metastability on chain folding, while short sequences will crystallize later, with a priority for melting on heating. The short sequences without the necessity of chain folding at the edge of lamellae, or in the granular crystallites, fringed micelles, or clusters, may pack quite loosely ${ }^{4}$ and contribute to pseudohexagonal crystallinity in ethylene-1-octene copolymers. ${ }^{60}$

However, in the case of our heterogeneous copolymers, the attained absolute crystallinity seems sensitive to the comonomer content. In association with the molecular segregation in the melt, this implies that the maximal probability of crystallization of the monomer sequences is only in homogeneous phases. If the crystallizable sequences are not homogeneously distributed in the melt, the monomer sequences, which are scarce in the comonomer-rich region, may not be able to form crystallites of the right size to reach stability through shortrange diffusion.

Any crystallinity or DSC curves produced in a temperature-scanning program can be regarded as the master curves of a statistical copolymer. These master curves just reflect the characteristic of sequence-length distribution on account of a local sequence-length segregation upon crystallization. The temperature function of equilibrium crystallinity, which can be approximately calculated from Flory-type theories, is not applicable to the master melting curve due to the kinetic reasons. ${ }^{11}$ On the experimental side, the master curves cannot be quantitatively reproduced by the parallel shifts of corresponding curves of fractions after fractionation, since their crystallizations may happen with different kinetics due to the difference in sequencelength distributions.

Figures $2 \mathrm{~b}$ and $4 \mathrm{a}$ show cold crystallization on heating if the crystallization on cooling has started but not yet completed. In our simulation systems, the crystallization on cooling is generated by homogeneous primary nucleation, while subsequent crystal growth needs less supercooling than the primary nucleation. If the lateral crystal growth of lamellae has not been completed yet at the starting point of heating due to a fast cooling, the lateral crystal growth will continue in the early stage of heating. This could be the reason cold crystallization occurs, and reflects the irreversible nature of phase transitions. Since the crystallite thickness depends on both the crystallization temperature and the monomer-sequence length, overgrowth on the preexisting lamellae gives rise to heterogeneity of thickness in the same lamellae. The short monomer sequences tend to perform crystal growth without chain folding, so the crystal thickness on overgrowth is expected to decrease quite fast due to surface crowding of the dangl ing ends connecting with short monomer sequences. Actually, experimental observations on LDPE have suggested an insertion mode of secondary crystallization without primary nucl eation, ${ }^{61}$ al though it is difficult to separate the primary crystallization from conventional secondary crystallization due to the incompleteness of primary crystal growth. This insertion mode means that the overgrowth on the lateral sides of lamellae occurs with decreasing thickness and higher content of short monomer sequences between two lamellae and ther efore be applicable to the present case.

The crystallinity gain in cold crystallization may have little association with the new thin lamellae formed in the interval of the stacked lamellae, 62 since the latter still needs primary nucleation, which is very sensitive to the temperature and will be suppressed on heating.

Naturally, the insertion mode could also happen on cooling. This implies that the crystallinity gain at lower temperatures is not always attributed to the granular domains, but can also be the overgrowth of the lamellae. In other words, sequence-length selection can happen not only in the generation of new crystallites but also in further growth of crystallites already present. The insertion mode is also applicable to explain the significant crystallinity gain after primary crystallization of ethylene-1-octene copolymers. ${ }^{63}$

Long-term isothermal annealing at a crystallization temperature facilitates further crystal growth and perfection of the present crystallites by the continued sequence selection. This can optimize poor crystallographic structures of lamellar overgrowth and granular domains to a limited extent, as revealed by Figure $4 \mathrm{~b}$ and DSC measurements. ${ }^{39,41}$ The melting of this part of optimized crystallites can be accumulated at slightly higher temperatures, leading to the so-called low endotherm. The low endotherm is just a local perturbation of the master curves both for crystallization and for melting, so it may not be attributed to the amorphous entropy loss only; ${ }^{39}$ otherwise the subsequent cooling curves at lower temperatures, away from the annealing temperature, should show a dependence on the annealing time rather than a convergent tendency. ${ }^{41}$ Another evidence of crystallite optimization is the decrease of reversing heat capacity on annealing ${ }^{41}$ For crystalline homopolymers, the significant excess heat capacity over the baseline (vibrational) heat capacity can be mainly attributed to the reversible breathing (melting/crystallization) at the fold surface of polymer crystallites as proved by temperature-modulated DSC and X-ray diffractions. ${ }^{64-66}$ For copolymer systems, this kind of reversible melting still exists and be probably more significant due to the small sizes of crystallites and poor crystallographic structures, which favor chain sliding diffusion. The decay of excess heat capacity as a function of time should be attributed to the optimization of crystallographic structure, especially near the fold surface, and without any increase in the lamellar thickness. ${ }^{35}$ The excess heat capacity appears fully reversible under temperature modulations and seems not to be associated with the fully melting and recrystallization of granular domains, ${ }^{60}$ which should show a hysteresis since their sizes are larger than the critical nucleus during primary nucleation. Actually, this hysteresis has been observed in the low-temperature regions of Figures 2 and 4.

In the crystallization of homogeneous copolymers accompanied by sequence segregation, long sequences will crystallize with chain folding while short sequences will directly confine the size of crystallites. This is the reason the thickness of crystallites is usually in the nanoscale, and it demonstrates the concept of nanophase separation driven by the crystallization of monomer 
sequences. ${ }^{67} \mathrm{~F}$ or the homogeneous copolymers with high comonomer content, the comonomers contribute to a continuous soft amorphous matrix while most of the monomers contribute to small rigid crystall ine domains. Long-chain molecules may pass through both phases several times. Therefore, the crystalline domains can act as physical cross-links under deformation. Actually, it reflects a typical assembling structure of thermoplastic elastomers and explains why homogeneous copolymers usually show high elasticity, as is the case with low-crystallinity ethylene-1-octene copolymers. ${ }^{68}$

The master crystallinity curves as a function of temperature for melting and crystallization can play a role as a phase diagram in fractionation analysis, and hence be useful to clarify the principles of temperature rising elution fractionation (TREF), ${ }^{69,70}$ crystallization analysis fractionation (CRYSTAF), ${ }^{71}$ and other fractionation methods based on crystallization/dissolution. ${ }^{1,23}$ TREF has been widely used to analyze the comonomer distribution within the molecules and to prepare copolymer fractions with narrow-distributed comonomer contents. In this method, copolymers crystallize very slowly on a substrate from the solution, by which the sequence selection takes place during crystal growth. Molecules containing the longest monomer sequences will precipitate in priority, while on subsequent dissolution, the reverse process takes place: Molecules containing the shortest monomer sequences will be dissolved first. It should be realized, however, that short monomer sequences will only be released when the longest monomer sequences in the same molecule dissolve, therefore, crystallization/dissolution methods should perform better in the cases of intermolecular heterogeneity and worse in the cases of intramolecular heterogeneity. ${ }^{1}$ For the latter cases, the analysis of DSC fractionation is more applicable. ${ }^{72}$

The heterogeneous copolymers can be regarded as a blend of copolymer molecules with quite different comonomer content and sequence distributions, see Figure $1 \mathrm{~b}$, and the molecular segregation before crystallization has actually been regarded as a liquid-liquid-phase separation in the blend. ${ }^{73-79}$ Since we have set the mixing exchange of the potential energy between the pairs of monomers and comonomers as zero, the driving force for the observed liquid-liquid-phase separation should be attributed to the molecular difference in crystallizable monomer content, which leads to a difference of molecular interactions in the melt. On this interesting topic, we will report further results and discussion later.

\section{Conclusions}

Many experimental observations about crystallization and melting of bulk statistical copolymers have been well reproduced by the simulations with a simple model taking into account of a packing-energy difference between monomer bonds and bonds containing comonomers and a sliding-diffusion restriction with respect to comonomers in the crystalline regions. Much more insights were then obtained. These include the following:

1. By comparison of the bulk phase transitions of three typical statistical copolymers, we demonstrated that the sequence distribution is also a major independent variable for the description of structure-property relationships of copolymers besi des the backbone chain length, the chemical nature of comonomers, and the comonomer content.
2. For statistically homogeneous copolymers, the phase-transition temperature, the final relative crystallinity, and the crystal morphology are very sensitive to the comonomer content as well as their distribution within the molecules, but the saturated absolute crystallinities of monomer sequences seem not to be sensitive to these parameters. The latter is in accord with strong sequence segregation during crystallization, or in other words, nanophase separation driven by crystallization of monomer sequences. This intramolecular segregation leads to assembling structures as in typical thermoplastic el astomers.

3. The master curves corresponding to crystallization and melting of a statistical copolymer were suggested on account of a local sequence-length segregation during crystallization. The kinetic effects of phase transitions of copolymers and the principle of temperature rising elusion fractionation and other fractionation methods can be understood based upon the master curves governed by the sequence-length distribution.

4. If the heating is performed before completeness of crystallization on cooling, cold crystallization can be observed. This reflects the irreversible nature of phase transitions of bulk copolymers and can be explained by an insertion mode of polymer crystal growth.

5. A liquid-liquid phase separation is observed in the melt of heterogeneous copolymers. The driving force of intermolecular segregation should be attributed to the significant difference in the content of crystallizable monomers between the copolymer molecules. This segregation is slightly enhanced by the hard restriction of sliding diffusion for comonomers in the crystalline regions.

Acknowledgment. W.H. expresses his thanks to Mr. F. G. Karssenberg at the DPI for the helpful discussions. This work was financially supported by DSM Co. The work of the FOM Institute is part of the research program of FOM and is made possible by financial support from the NWO.

\section{References and Notes}

(1) Mathot, V. B. F. In Calorimetry and Thermal Analysis of Polymers; Mathot, V. B. F., Ed.; Hanser Publishers: Munich, Germany, 1994; Chapter 9.

(2) Alamo, R. G.; Mandelkern, L. Thermochim. Acta 1994, 238, 155.

(3) Mathot, V. B. F.; Scherrenberg, R. L.; Pijpers, T. F. J .; Engelen, Y. M. T. In New Trends in Polyolefin Science and Technology; Hosoda, S., Ed.; Research Signpost: Trivandrum, India, 1996; p 71.

(4) van Ruiten, J .; van Dieren, F.; Mathot, V. B. F. In Crystallization of Polymers; Dosiere, M., Ed.; NATO ASI-C Series 405 on Mathematical and Physical Science; 1993; p 481.

(5) Hu, W.-B. J . Chem. Phys. 2000, 113, 3901.

(6) Flory, P. J. J . Chem. Phys. 1947, 15, 684.

(7) Flory, P. J . Trans. Faraday Soc. 1955, 51, 848.

(8) Helfand, E.; Lauritzen, J . I., J r. Macromolecules 1973, 6, 631.

(9) Sanchez, I. C.; Eby, R. K. Macromolecules 1975, 8, 638.

(10) Wunderlich, B. Macromolecular Physics; Academic Press: New York, 1980; Vol. 3, Crystal Melting, 264.

(11) Crist, B.; Howard, P. R. Macromolecules 1999, 32, 3057.

(12) Vanden Eynde, S.; Rastogi, S.; Mathot, V. B. F. Macromolecules 2000, 33, 9696.

(13) Vanden Eynde, S.; Mathot, V. B. F.; Hoehne, G. W. H.; Schawe, J. W. K.; Reynaers, H. Polymer 2000, 41, 3411.

(14) Alamo, R. G.; Mandelkern, L. Macromolecules 1991, 24, 6480.

(15) Hu, W.-B. J . Chem. Phys. 1998, 109, 3686.

(16) IUPAC, Pure Appl. Chem. 1985, 57, 1427.

(17) Alfrey, T. J r.; Bohrer, J. J .; Mark, H. Copolymerization; Interscience: New York, 1952.

(18) Flory, P. J . Principles of Polymer Chemistry; Cornell University Press: Ithaca, NY, 1953; Chapter 5. 
(19) Ham, G. E. In Copolymerization; Ham, G. E., Ed.; Interscience: New York, 1964

(20) Cowie, J. M. G. Polymers: Chemistry and Physics of Modern Materials; Blackie and Son: Glasgow, Scotland, and London, 1991; Chapter 5.

(21) Coote, M. L.; Davis, T. P. Prog. Polym. Sci. 1999, 24, 1217.

(22) Madkour, T. M.; Goderis, B.; Mathot, V. B. F.; Reynaers, H. Polymer 2002, 43, 2897.

(23) Mathot, V. B. F. Polycon'84 LLDPE; Plast. Rubber Inst., Chameleon Press: London, 1984; $p 1$.

(24) Schouterden, P.; Groeninckx, G.; van der Heyden, B.; J ansen, F. Polymer 1987, 28, 2099.

(25) Hosoda, S. Polym. J . 1988, 20, 383.

(26) Karbashewski, E.; Kale, L.; Rudin, A.; Tchir, W. J .; Cook, D. G.; Pronovost, J. O. J. Appl. Polym. Sci. 1992, 44, 425.

(27) $\mathrm{Hu}$, W.-B.; Mathot, V. B. F.; Frenkel, D. Manuscript in preparation.

(28) Larson, R. G.; Scriven, L. E.; Davis, H. T. J . Chem. Phys. 1985, 83, 2411.

(29) Camesin, I.; Kremer, K. Macromolecules 1988, 21, 2819.

(30) Deutsch, H. P.; Binder, K. J . Chem. Phys. 1991, 94, 2294.

(31) Lu, J .-M.; Yang, Y.-L. Sci. Chin. Ser. A 1993, 36, 357.

(32) de Gennes, P.-G. J . Chem. Phys. 1971, 55, 571.

(33) Hu, W.-B. J . Chem. Phys. 2001, 115, 4395.

(34) van den Eynde, S.; Mathot, V. B. F.; Koch, M. H.J .; Reynaers, K. H. Polymer 2000, 41, 4889.

(35) Hauser, G.; Schmidtke, J .; Strobl, G. Macromolecules 1998, $31,6250$.

(36) Mathot, V. B. F.; Scherrenberg, R. L.; Pijpers, T. F. J . Polymer 1998, 39, 4541.

(37) Kilian, H. G.; Fischer, E. W. Kolloid Z. Z. Polym. 1966, 211, 40.

(38) Bensason, S.; Minick, J.; Moet, A.; Chum, S.; Hiltner, A.; Baer, E. J . Polym. Sci., Part B: Polym. Phys. 1996, 34, 1301.

(39) Alizadeh, A.; Richardson, L.; Xu, J .; McCartney, S.; Marand, H.; Cheung, Y. W.; Chum, S. Macromol ecules 1999, 32, 6221.

(40) Zhang, F.J.; Liu, J. P.; Xie, F. C.; Fu, Q.; He, T. B. J . Polym. Sci., Part B: Polym. Phys. 2002, 40, 822.

(41) Androsch, R.; Wunderlich, B. Macromol ecules 1999, 32, 7238.

(42) Hanna, S.; Romo-Uribe, A.; Windle, A. H. Nature (London) 1993, 366, 546.

(43) Krigas, T.; Carella, J .; Struglinski, M.; Crist, B.; Graessley, W. W.; Schilling, F. C. J . Polym. Sci.: Polym. Phys. Ed. 1985, 23, 509.

(44) Mirabella, F. M.; Westphal, S. P.; Fernando, P. L.; Ford, E. A.; Williams, J. G. J . Polym. Sci., Part B: Polym. Phys. 1988, 26, 1995

(45) Kim, M.-H.; Phillips, P.J . J . Appl. Polym. Sci. 1998, 70, 1893.

(46) Gelfer, M. Y.; Winter, H. H. Macromolecules 1999, 32, 8974.

(47) Wignall, G. D.; Alamo, R. G.; Ritchson, E. J .; Mandelkern, L.; Schwahn, D. Macromolecules 2001, 34, 8160.

(48) Lambert, W. S.; Phillips, P. J . Polymer 1996, 37, 3585.

(49) McFaddin, D. C.; Russell, K. E.; Wu, G.; Heyding, R. D. J . Polym. Sci., Phys. 1993, 31, 175.

(50) Zhang, M.; Lynch, D. T.; Wanke, S. E. Polymer 2001, 42, 3067.

(51) Zhang, X.; Li, Z.; Lu, Z.; Sun, C J . Chem. Phys. 2001, 115, 3916.

(52) Balbontin, G.; Camurati, I.; Dall'Occo, T.; Finotti, A.; Franzese, R.; Vecellio, G. Angew. Macromol. Chem. 1994, 219, 139.
(53) Mirabella, F. M. J . Polym. Sci., Part B: Polym. Phys. 2001, 39, 2800.

(54) Goderis, B.; Peeters, M.; Mathot, V. B. F.; Koch, M. H. J .; Bras, W.; Ryan, A. J .; Reynaers, H. J . Polym. Sci., Part B: Polym. Phys. 2000, 38, 1975.

(55) Litvinov, V. M.; Mathot, V. B. F Solid State NMR 2002, 22, 218.

(56) Herrmann, K.; Gerngross, O.; Abitz, W. Z. Phys. Chem. 1930, $10,371$.

(57) Kilian, H. G. Thermochim. Acta 1994, 238, 113.

(58) Wunderlich, B. J . Chem. Phys. 1958, 29, 1395.

(59) Crist, B.; Claudio, E. S. Macromolecules 1999, 32, 8945.

(60) Androsch, R.; Wunderlich, B. Macromol ecules 2000, 33, 9076.

(61) Strobl, G.; Engelke, T.; Meier, H.; Urban, G. Colloid Polym. Sci. 1982, 260, 394 or Strobl, G. The Physics of Polymers; Springer: Berlin, 1997; p 184.

(62) Verma, R.; Marand, H.; Hiao, B. Macromolecules 1996, 29, 7767.

(63) Akpalu, Y.; Kielhorn, L.; Hsiao, B. S.; Stein, R. S.; Russell, T. P.; van Egmond, J .; Muthukumar, M. Macromolecules 1999, 32, 765 .

(64) Hu, W.-B.; Albrecht, T.; Strobl, G. Macromol ecules 1999, 32, 7548.

(65) Albrecht, T.; Armbruster, S.; Keller, T.; Strobl, G. Macromolecules 2001, 34, 8456.

(66) Goderis, B.; Reynaers, H.; Scherrenberg, R.; Mathot, V. B. F.; Koch, M. H. J . Macromolecules 2001, 34, 1779.

(67) Chen, W.; Wunderlich, B. Macromol. Chem. Phys. 1999, 200, 283.

(68) Bensason, S.; Stepanov, E. V.; Chum, S.; Hiltner, A.; Baer, E. Macromol ecules 1997, 30, 2436.

(69) Wild, L. Adv. Polym. Sci. 1990, 98, 1.

(70) Xu, J .-T.; Feng, L.-X. Eur. Polym. J . 2000, 36, 867.

(71) Monrabal, B. In New Trends in Polyolefin Science and Technol ogy; Hosoda, S., Ed.; Research Signpost: Trivandrum, India, 1996; p 119.

(72) Keating, M. Y.; McCord, E. F. Thermochim. Acta 1994, 243, 129.

(73) Mirabella, F. B.; Ford, E. A. J . Polym. Sci., Part B..; Polym. Phys. 1987, 25, 777.

(74) Deblieck, R. A. C.; Mathot, V. B. F. J . Mater. Sci., Lett. 1988, 7, 1276.

(75) Crist, B.; Hill, M. J . J . Polym. Sci.: Polym. Phys. Ed. 1997, 35, 2329.

(76) Alamo, R. G.; Graessley, W. W.; Krishnamoorti, R.; Lohse, D. J .; Londono, J . D.; Mandelkern, L.; Stehling, F. C.; Wignall, G. D. Macromolecules 1997, 30, 561.

(77) Fu, Q.; Chiu, F.-C.; McCreight, K. W.; Guo, M.; Tseng, W. W.; Cheng, S. Z. D.; Keating, M. Y.; Hsieh, E.; DesLauriers, P. J. J . Macromol. Sci.-Phys. 1997, B36, 41.

(78) Chen, F.; Shanks, R.; Amarasinghe, G. J . Appl. Polym. Sci. 2001, 81, 2227.

(79) Wang, H.; Shimizu, K.; Kim, H.; Hobbie, E. K.; Wang, Z.-G.; Han, C. C. J . Chem. Phys. 2002, 116, 7311.

MA0213854 